BULLETIN Bulletin hispanique

HISPANIQUE Université Michel de Montaigne Bordeaux

112-1| 2010

Actes du Colloque « langue, littérature, littéralité »

\title{
Bois d'Amour, jardins et château dans les Cigarrales de Toledo
}

Nathalie Dartai-Maranzana

\section{OpenEdition}

\section{Journals}

Édition électronique

URL : http://journals.openedition.org/bulletinhispanique/1118

DOI : 10.4000/bulletinhispanique.1118

ISSN : 1775-3821

Éditeur

Presses universitaires de Bordeaux

\section{Édition imprimée}

Date de publication : 1 juin 2010

Pagination : 131-147

ISBN : 978-2-86781-692-5

ISSN : 0007-4640

Référence électronique

Nathalie Dartai-Maranzana, "Bois d'Amour, jardins et château dans les Cigarrales de Toledo », Bulletin hispanique [En ligne], 112-1 | 2010, mis en ligne le 01 juin 2013, consulté le 03 mai 2019. URL : http:// journals.openedition.org/bulletinhispanique/1118; DOI : 10.4000/bulletinhispanique.1118 


\title{
Bois d'Amour, jardins et château dans les Cigarrales de Toledo
}

\author{
Nathalie Dartai-Maranzana \\ Université Lumière - Lyon 2
}

En hommage au travail de direction de ma thèse sur les Cigarrales de Toledo assuré par Nadine Ly, le présent article analyse l'allégorie et les emblèmes du château de verdure du Cigarral II pour montrer en quoi ce bois d'Amour ou ce château-jardin (le "Castillo de la Pretensión de Amor ") est la métonymie de la structure narrative et thématique des Cigarrales de Toledo de Tirso de Molina.

Para rendir homenaje a Nadine Ly, directora de mi tesis sobre los Cigarrales de Toledo, el presente artículo analiza la alegoría y los emblemas del castillo de vegetación del Cigarral II para mostrar en qué medida ese bosque de Amor o ese castillo-jardin (el "Castillo de la Pretensión de Amor ") es la metonimia de la estructura narrativa y temática de los Cigarrales de Toledo de Tirso de Molina.

This article, dedicated to Nadine Ly, with my heartfelt gratitude for her supervising my PhD dissertation on 'Cigarrales de Toledo', analyses the allegory and emblems linked with the motive of the castle of greenery in 'Cigarral II'. My aim is to demonstrate that this copse of Love, this castle-garden - the "Castillo de la Pretensión de Amor" - in fact constitutes a metonymy of the narrative and thematic structure of Tirso de Molina's 'Cigarrales de Toledo'.

Mots-clés : Allégorie - Emblème - Cigarrales - Structure.

$B H i$, Tome 112, no 1 - juin 2010 - p. 131 à 147. 
L

E CHOIX du passage des Cigarrales de Toledo que je me propose d'étudier ici à l'occasion du colloque hommage à Nadine Ly s'est imposé en quelque sorte de lui-même. Quand je pense à Nadine Ly, c'est bien sûr à la directrice de thèse attentive et critique qu'elle a été pour moi et aussi au professeur qui m’a révélé les méandres et les arcanes allégoriques du Criticón de Gracián lorsque j'ai préparé l'agrégation externe à l'université de Bordeaux 3. C'est donc en hommage à son travail de direction de ma thèse sur les Cigarrales de Toledo et en souvenir de ses cours captivants sur l'allégorique Criticón que j'ai eu envie de revenir aux sources et de m'intéresser à un extrait du cigarral II que je n'avais pas analysé dans le détail pour ma thèse et qui peut être considéré comme un petit joyau de littérature emblématique et allégorique.

Les Cigarrales de Toledo se présentent comme une œuvre miscellanée constituée de cinq cigarrales qui sont autant de chapitres de l'œuvre et qui sont précédés d'une très longue introduction mettant en place tous les ingrédients narratifs, fictionnels, thématiques et structurants de l'œuvre. Le passage qui m’intéresse ici se situe au début du cigarral II (p. 233-259 de l'édition Castalia, 1996 ${ }^{1}$ ) alors que les personnages du récit-cadre ont quitté le premier cigarral, celui de Buenavista où ils ont assisté, entre autres choses, à la représentation de la comedia El vergonzoso en palacio, pour arriver au cigarral de don Gaspar de Quiroga. En vertu d'une règle qui a été édictée au départ par les jeunes nobles tolédans qui ont décidé de se divertir dans les propriétés et jardins des hauteurs de Tolède pour échapper à la canicule estivale, la belle Narcisa a été désignée "reine " de ce cigarral et par conséquent aussi, organisatrice des divertissements qui doivent se tenir en ce lieu et au cours de ce chapitre II. C'est donc le premier de ces divertissements qui est évoqué longuement dans notre passage. On pourrait l'appeler le divertissement du "Castillo de la Pretensión de Amor» en référence à la p. 236 mais ce nom ne dit pas toute la complexité du jeu proposé par Narcisa. En effet, la première partie du passage est une longue introduction (p. 233-237) qui nous présente l'entrée du Bois d'Amour, l'accueil des invités au cigarral et au Bois d'Amour par un bel enfant, par la reine Narcisa et un petit orchestre avec lequel l'enfant - qui est l'allégorie du Plaisir ( $u n$ hermoso niño, figurado en él el Placer» p. 234) - et des musiciens chantent une Letra. Puis les règles du jeu sont indiquées aux hôtes de Narcisa : les dames promises aux six galants qui vont entreprendre la conquête du "Château de

1. Tirso de Molina, Cigarrales de Toledo, Madrid, Clásicos Castalia, $\mathrm{n}^{\circ} 216$, édition de Luis Vázquez Fernández, 1996. Toutes les références au texte de Tirso seront tirées de cette édition. 
la Prétention d'Amour $»^{2}$, Narcisa, les anciens et les veufs entrent par l'arche principale du Bois d'Amour qui se situe devant le Château. Narcisa devient ainsi la geôlière des dames célibataires et les galants vont devoir les libérer. La deuxième partie du passage (p. 237-256) évoque alors le Bois d'Amour que le texte désigne aussi comme "la simbólica Selva» (p. 256). Après s'être séparés en deux groupes (p. 237-239), les galants aventuriers entrent dans le Bois d'Amour par les deux arches encadrant la porte principale. Don Fernando, don Melchor et don Vela, les méfiants, entrent par la porte du doute (" el arco de la sospecha ») et connaissent diverses aventures (p. 239-246) ; don Miguel, don Suero et don Alonso pénètrent dans le Bois d'Amour par la porte de la confiance ("la puerta de la confianza ") et arrivent au Château après maintes péripéties (p. 246-256). Enfin, dans la troisième et dernière partie du passage (p. 256-259), nous assistons à l'attaque du château par les six "prétendants d'amour ", puis à son effondrement et enfin à un Banquet somptueux qui précède une sieste bien gagnée et se présente donc comme la conclusion du divertissement. On remarque par conséquent que la partie centrale du passage est la plus longue et met l'accent sur le Bois d'Amour qui est un véritable labyrinthe semé d'embûches, de figures allégoriques et d'emblèmes destinés aux jeunes nobles qui prétendent à l'amour de leurs dames enfermées dans le "Château de la Prétention d'Amour ». D'ailleurs, la longue phrase qui introduit le passage et que Tirso de Molina n'aura de cesse de développer, amplifier, n'évoque que ce "labyrinthe d'arbres et de fleurs ». Je n'en cite qu'une partie, la plus importante et celle que je considère comme la phrase-clé de tout le passage (le sujet est Narcisa) p. 233 :

No se habia descuidado la solícita dama, pues [...] habia, en la entrada del celebrado Cigarral, dispuesto un enramado laberinto de árboles y flores, con diferentes calles y lazos, tan imitador del de Creta, que aunque faltó el monstruoso parto de Pasife no a lo menos el ingenio de Dédalo ni la confusión de Teseo.

Tout est dit : en se référant au minotaure mythologique de Crète qui hantait les allées du labyrinthe conçu par Dédale et dans lequel Thésée l'affronta et le tua, Tirso de Molina place le divertissement de Narcisa, comparée ici à Dédale, sous le signe du mystère allégorique et emblématique, sous le signe de l'art et de l'artifice (ingenio), sous celui de l'amour, de la mort et de l'aventure, sous le signe enfin du parcours initiatique. Il nous donne aussi les clés de lecture de ce passage qui est placé également, ne l'oublions pas, sous le signe du plaisir et de la fête par la Letra que chante la figure allégorique

2. Le texte les qualifie de «los comprehendidos en la amorosa pretensión». 
du Plaisir. Par conséquent, je me contenterai de le suivre en analysant tour à tour les grandes caractéristiques de ce texte : le labyrinthe d'arbres et de fleurs, le divertissement et le plaisir, l'amour emblématisé que je réunirai en deux grandes parties : le labyrinthe floral, emblème de la structure des Cigarrales de Toledo et le jeu du labyrinthe, emblème des thématiques de l'œuvre tirsienne. Entrons donc sans plus attendre dans ce labyrinthe pour en découvrir les secrets et surtout la fonction dans toute l'œuvre.

\section{LE LABYRINTHE FLORAL,} EMBLÈME DE LA STRUCTURE DE L'CEUVRE

Si, comme je l'ai montré dans ma thèse ${ }^{3}$, le motif du jardin est essentiel dans l'œuvre tirsienne tant sur le plan de l'espace que sur le plan de la structure, nous nous trouvons ici, dans ce deuxième chapitre, en présence d'un jardin exubérant, construit ingénieusement et artificiellement (cf. p. 234 : " el artificioso bosque que, para dar principio a su fiesta, habia hecho plantar la industriosa Narcisa ") à l'intérieur de l'espace-jardin du cigarral de don Gaspar de Quiroga, en présence donc d'un labyrinthe floral enchâssé, d'un cigarral démultiplié ou hypertrophié.

\section{I.1 Le « cigarral ", espace-jardin, hypertrophié}

En effet, on peut considérer que le "labyrinthe d'arbres et de fleurs " (cf. p. 233) qu'est le Bois d'Amour du divertissement proposé par Narcisa est l'image amplifiée de tous les cigarrales de l'œuvre tirsienne où séjournent les personnages et qui sont à la fois des espaces du récit-cadre et des espaces de récits (puisque selon le modèle boccacien du Décameron, des personnages racontent des histoires, bien souvent leurs propres pérégrinations, dans plusieurs chapitres). Il est le jardin par excellence car la multiplicité des essences d'arbres et des variétés de fleurs qui le composent lui donne un aspect foisonnant qui rappelle et en même temps démultiplie la végétation décrite au début de chaque cigarral. Ainsi, on ne compte pas moins de 13 occurrences des mots flores et florido ou florida dans les pages consacrées au Bois d'Amour et au château, occurrences qui sont la plupart du temps associées à l'idée d'abondance et de diversité. La liste des arbres (álamo, ciprés,

3. Les "Cigarrales de Toledo": recherches sur l'architecture d'un "jardin ", Université Michel de Montaigne-Bordeaux 3, 1996 (thèse publiée aux Presses Universitaires du Septentrion en 1998). 
moral, melocotones, naranjos, manzanos, pinos, palmera etc.), des arbustes (laurel, tarayes, enebros, arrayán, murta, nuezas etc.), des plantes aromatiques (romeros, albahacas etc.), des mauvaises herbes (hortigas, hierbas, malezas, zarzas etc.) et des fleurs enfin (jazmines, rosas, lirios, clavellinas, azucenas, adormideras etc.) comporte 61 termes. Ainsi, cette abondance lexicale des mots ayant trait à la végétation souligne l'insistance du texte tirsien sur la profusion florale et arboricole et, de fait, sur le motif du jardin. Ce n'est sans doute pas un hasard non plus si la reine de ce cigarral, l'inventeur ingénieux de ce labyrinthe floral et l'organisatrice de ce divertissement, se nomme Narcisa. On sait que le narcisse est une plante à fleurs campanulées très odorantes, blanches ou jaunes et que, symboliquement, dans la Bible, il caractérise le printemps, saison par excellence de l'exubérance végétale et de la renaissance de la nature. Ainsi, Tirso, grâce au jeu onomastique, fait de Narcisa la figure emblématique du jardin labyrinthique qu'elle fait disposer dans le cigarral de don Gaspar de Quiroga. D'ailleurs, pour couronner le tout, si je puis dire, Narcisa est présentée (p. 234) couronnée d'une guirlande de violettes et d'œillets.

Car il s'agit bien d'un véritable labyrinthe qui se réclame, nous l'avons vu déjà, du labyrinthe crétois de Dédale (p. 233) et qui répond au goût et à l'art baroques des architectures éphémères (il est question de su florida arquitectura à la p. 241) et des dédales de buissons et arbustes conçus dans le seul but de divertir les visiteurs des jardins ou des parcs. En effet, à l'exception du chemin emprunté par don Miguel, toutes les allées de ce labyrinthe floral se subdivisent en deux, trois, ou plusieurs voies. Ainsi, don Suero doit littéralement choisir entre deux chemins indiqués par une main sur laquelle est écrit " escoge "; don Alonso se retrouve devant trois voies et, incité par l'impératif "Guiete tu confianza ", choisit de prendre l'allée de l'Absence; du côté des soupçonneux et des méfiants, don Fernando est amené à prendre le chemin appelé "Por aqui " parmi six possibilités qui s'offrent à lui ; don Melchor doit choisir entre trois voies et emprunte celle qui le conduira à la figure allégorique de la Persévérance ; enfin don Vela se trouve, lui aussi, devant plusieurs chemins : il en emprunte tour à tour un certain nombre mais revient sans cesse à son point de départ, pris au piège de voies sans issue. De plus, tous les "prétendants d'amour » se retrouvent sur une place devant le château, lieu qui représente le centre de tout labyrinthe si l'on reprend la définition que donne le Dictionnaire des symboles de Jean Chevalier et Alain Gheerbrant ${ }^{4}$ : «le labyrinthe est essentiellement un

4. Jean Chevalier et Alain Gheerbrant, Dictionnaire des symboles, Robert Laffont, 1969, édition revue et corrigée, 1982. 
entrecroisement de chemins dont certains sont sans issue, à travers lesquels il s'agit de découvrir la route qui conduit au centre de cette bizarre toile d'araignée ». Enfin, l'enchevêtrement des chemins et de la végétation de ce labyrinthe de Narcisa (plusieurs expressions disent cet enchevêtrement telles que "florida confusión" p. 234, "intrincada calle " p. 241, " un enramado laberinto" p. 233, "la enmarañada confusión" p. 239 etc.) est l'emblème de la miscellanée tirsienne qui mêle savamment les récits (de niveau principal et secondaire), les genres (pièces de théâtre, poèmes et nouvelle) et entraîne le lecteur dans les méandres d'histoires complexes au travers de ses cinq cigarrales. D'ailleurs, si dans la première partie de ma thèse (chapitre 2), j'ai évoqué "la construction arboriforme de l'œuvre " ${ }^{5}$, il est frappant de constater que les différents chemins du labyrinthe de Narcisa dessinent la forme de deux arbres majestueux à trois branches dont le sommet serait le "Castillo de la Pretensión de Amor" et le tronc, la porte de chaque entrée à droite et à gauche du labyrinthe. Ainsi, le labyrinthe floral du cigarral II serait l'emblème de toute l'œuvre intitulée Cigarrales de Toledo et, en particulier, la métonymie de sa structure. Et je prends le terme "emblème " dans son sens le plus littéral, de sorte que le labyrinthe floral et le Château seraient la partie figurative et centrale de l'emblème, le titre « Castillo de la Pretensión de Amor ", la devise et les quelques lignes de la "premática ${ }^{6}$ " p. 236, la légende explicative de l'emblème en même temps que l'explication des règles du jeu.

Mais le labyrinthe de Narcisa est aussi conçu comme un parcours initiatique.

\section{I.2 Le labyrinthe comme parcours initiatique}

En effet, suivant en cela la symbolique du labyrinthe qui veut que, je cite encore le Dictionnaire des symboles, " le labyrinthe [doive] à la fois permettre l'accès au centre par une sorte de voyage initiatique et l'interdire à ceux qui ne sont pas qualifiés ", Tirso de Molina fait de chaque cheminement des

5. «La construction de l'œuvre s'apparente en effet à un arbre dans la mesure où le titre amplifié comme nous l'avons vu précédemment, par la série des cinq cigarrales, s'amplifie encore sous la forme de trois branches ou ramifications : les évocations de jardins au début des cigarrales, les divertissements et les passages de critique littéraire à la fin de chaque cigarral. Il y a donc parfaite adéquation entre l'architecture de l'œuvre et sa structure sémantique et cette construction que je qualifie d'arboriforme éclaire après coup le sens total du titre ; il est porteur du contenu (poétique tirsienne) et de l'architecture de l'œuvre : le jardin est texte et le texte est jardin."

6. Le Tesoro de Covarrubias définit ce terme ainsi : "La ley que se promulga, en razón de las nuevas ocasiones que se ofrecen en la república para remediar excesos y daños». 
participants au divertissement de Narcisa, un apprentissage, un parcours initiatique qui va donner une leçon à chacun d'eux et le transformer pour lui donner accès au centre, cette place sur laquelle débouchent les voies du labyrinthe floral et ce Château d'Amour où sont enfermées les dames, et pour lui permettre d'atteindre la félicité amoureuse. Ainsi, seuls les initiés aux choses de l'amour entrent par la grande porte centrale du labyrinthe floral : les veufs, les sages anciens, la reine du cigarral et les dames qui attendent d'être conquises par leurs jeunes amants. C'est ce qu'indique la "premática" en lettres dorées figurant au-dessus de cette porte:

Al CASTILLO DE LA PRETENSIÓN DE AMOR ninguno se atreva a entrar por esta puerta, que sólo se franquea para los que, estando en posesión, tiran con apacibles coyundas el triunfo de Himeneo, y para las damas que, en compañia de nuestra Reina en la fortaleza defendida, fian del valor de sus amantes la libertad de su entretenido cautiverio (p. 236).

Pour les non initiés, les portes latérales s'ouvrent sur les allées du labyrinthe qu'ils vont devoir parcourir pour parvenir au château. Or ces allées sont pavées d'emblèmes et de figures allégoriques qui guident les pas des aventuriers et leur donnent des enseignements moraux. Par ailleurs, on se souvient que l'emblème a une fonction essentiellement morale comme le rappelle Christian Bouzy dans son article : "L'emblématique des lieux communs dans La Dorotea " ${ }^{7}$. C'est tout à fait le cas ici puisque les galants qui manquent de confiance, doutent de leurs bien-aimées, sont jaloux, entrent par la porte de droite (" el arco de la sospecha») et vont devoir apprendre à persévérer et à avoir confiance pour obtenir l'amour inconditionnel de leurs dames. Au contraire, ceux qui ont une confiance excessive entrent dans le labyrinthe par la porte de gauche ("la puerta de la confianza») et vont devoir apprendre à se méfier, à être prudents et vigilants. Je ne prendrai que deux exemples car le temps qui m'est imparti ne me permet pas d'analyser tous les parcours. Du côté droit, don Fernando, emprunte le chemin de la crainte et, comme l'y invitent trois vers, s'asseoit sur une chaise de fleurs qui, par la symbolique de la fleur ${ }^{8}$ et l'inscription au dos de la chaise ("Esperanza"), représente l'espoir vers lequel doit tendre au contraire le craintif prétendant ; puis il choisit parmi les six voies qui s'offrent à lui et figurent les effets de

7. Christian Bouzy, «L'emblématique des lieux communs dans La Dorotea : Lope de Vega dans l'entre-deux ", Lectures d'une auvre : La Dorotea, collectif coordonné par Nadine Ly, Éditions du Temps, 2001, p. 65-123.

8. Cf. Maurice Pillard-Verneuil, Dictionnaire des symboles, emblèmes et attributs, Genève, Slatkine Reprints, "Collection Ressources 112 », 1981 et Dictionnaire des symboles, ed. cit. 
la crainte (Désespoir, Vengeance, Colère, Courroux, Impatience), celle du milieu qui ne présente aucun effet de la crainte, précisément pour s'en éloigner et apprendre à être confiant et plein d'espoir : "Por aquí». Cette voie médiane le conduit à une placette fleurie où se trouve un laurier sans feuilles mais portant quelques bourgeons annonciateurs des récompenses que don Fernando peut espérer. Sur le tronc de ce laurier est écrit : "Aún no es tiempo ", signifiant par là à don Fernando qu'il faut persévérer comme le confirme la suite du parcours. En effet, le chemin est ensuite bordé de "flores en esperanza", de roses et de fruits auxquels se mêlent des écriteaux : "Poco a poco ", "Hasta el fruto " et il débouche sur un mûrier couvert de fruits et portant l'inscription "Para ti ». Sur l'écorce du mûrier figurent les vers suivants : "A quien cual yo con paciencia / y esperanzas se sazona, / ila posesión le corona!» (p. 241), message moral s'il en est de persévérance, de patience et d'espoir qui vient couronner la cohérence symbolique de l'ensemble. Le deuxième exemple, je le prends du côté gauche, celui des trop confiants. Don Suero, présenté tout de suite comme prétentieux par le narrateur ${ }^{9}$, a emprunté le chemin de la "estimación de sus servicios" et est tombé sur la figure de la connaissance de soi, qui lui rappelle qu'il n'est qu'un homme (Memento homo lui signifie un parchemin peint accroché à l'un des bras de la figure); puis il a emprunté à droite une des deux voies qui s'offraient à lui pour y voir des roseaux verts dont les feuilles portent l'inscription : «Vanidad de pensamientos" et parvenir à une table sur laquelle est représenté le monde surmonté d'une demi-lune argentée. Incité par une carte à découvrir cette énigme, il ne fait que trouver une cage à oiseaux et libérer ces oiseaux qui vont se moquer de sa vaine présomption. Puis il traverse une pièce de terre, désignée comme une "sala alegórica " dont les parois de verdure sont remplies de miroirs, symbole du "Desengaño" pour parvenir à quelques marches qui le conduisent au sommet d'un rocher. Là, un « char triomphant " l'attend et à peine est-il monté dans ce "Carro del Sol» qu'il fait une terrible chute, tel un nouveau Phaéton. Les vers qu'il trouve à ses pieds, une fois remis de sa chute, ("Cayó Faetón presumido; / ipero en ti no será afrenta / como caigas en la cuenta! ») finissent d'éclairer le sens moral du parcours du présomptueux et trop confiant don Suero.

Les deux exemples que je viens de donner et de décrire appellent quelques commentaires. Chaque cheminement est fondé sur l'antithèse, et la métamorphose intellectuelle et morale qu'il impose par ses emblèmes fait passer le concurrent d'un état donné à son contraire, d'un défaut à une qualité. Il y a bien apprentissage ou prise de conscience comme l'indiquent

9. «El, en fin, (si no soberbio, presumido) entró por la sobredicha senda...» (p. 250). 
d'ailleurs les qualificatifs employés pour désigner les participants au jeu de Narcisa : par exemple, "el escarmentado galán » (p. 249); et cet apprentissage se fait par les enseignements des emblèmes qui jalonnent les allées du labyrinthe floral. Car le texte ne cesse de mettre en évidence son utilisation d'emblèmes floraux par les termes de "letra ", "membrete ", avisos. Ainsi, chaque figure rencontrée est un véritable emblème dont on retrouve les trois grandes parties constitutives. Reprenons l'exemple du laurier sans feuilles du parcours de don Fernando (p. 240) : il est évident que l'arbuste constitue l'image ou la partie iconographique et centrale de l'emblème, la phrase "Aún no es tiempo ", sa devise et les trois vers sa légende explicative. Il en est de même pour la chaise fleurie sur laquelle don Fernando s'asseoit : la chaise composée de fleurs est l'image de l'emblème, le mot «Esperanza » écrit sur le dossier est la devise ou le titre et les trois vers en bas, la légende, l'explication. Par ailleurs, les symboles qu'utilise Tirso ne sont pas choisis au hasard mais s'intègrent dans une parfaite cohérence à l'allégorie générale et démonstrative. Ainsi, tous les emblèmes et les symboles du parcours du présomptueux don Suero sont en relation avec la thématique de la vanité humaine et la volonté de puissance ou de domination. Les uns disent cette thématique : les verts roseaux symbolisant la vacuité et la légèreté, la table surmontée d'un globe terrestre et d'une demi-lune argentée, le rocher (p. 252) qui symbolisent le désir de domination, le char du soleil qui rappelle l'entreprise insensée de Phaéton dans son désir d'égaler la puissance divine de son père le Soleil. Les autres au contraire la mettent à mal : la figure de la connaissance de soi qui tient dans sa main gauche un plat de cendres et dans l'autre, entre le pouce et l'index, de la cendre qu'elle laisse s'écouler, rappelant à l'homme qu'il est mortel et qu'il ne doit pas l'oublier (p. 250), les miroirs qui renvoient à don Suero une image très laide ou monstrueuse (p. 252) pour lui ouvrir les yeux sur sa vaine présomption ${ }^{10}$. De cette antithèse naît le message moral de la série d'emblèmes qui jalonnent le parcours initiatique de don Suero : celui-ci doit perdre sa vanité, sa trop grande assurance pour revenir à de plus justes et plus saines perceptions des choses, s'il veut atteindre le bonheur amoureux. Pour don Fernando, tous les emblèmes choisis disent l'espoir et la promesse de récompenses et viennent ainsi contredire le nom du sentier emprunté par le pusillanime personnage ("Temor»). Ainsi, la chaise de fleurs, le laurier sans feuilles puisque le laurier est l'emblème de la gloire et est associé à la récompense, à la victoire et à la persévérance, le mûrier

10. Rappelons que le miroir reflète la vérité, la sincérité, le contenu du cœur et de la conscience ; il est le symbole de la sagesse et de la connaissance. Il est aussi l'attribut de Luxuria (la luxure, la vanité) et symbolise les vertus de la connaissance de soi : veritas (vérité) et prudentia (prudence) (cf. Dictionnaire des symboles, ed. cit.). 
enfin, dont on sait qu'il tarde longtemps à développer ses feuilles ${ }^{11}$, qui est le symbole de la sagesse et est aussi associé à l'histoire tragique des deux amants mythologiques, Pyrame et Thisbée.

Enfin, on remarque que les emblèmes des différents parcours, ceux de don Fernando et de don Suero mais aussi ceux de don Miguel (le lit de la confiance tout en fleurs, la Vigilance sur un autel tâchant de protéger une femme en verre contre divers adversaires : "Ocasiones ", "Dádivas ", "Diligencias» p. 249), de don Melchor (la Persévérance qui tient par la main Cupidon, lequel porte dans la sienne une couronne de chêne p. 242), de don Vela et de don Alonso (par exemple l'éléphant qui a une petite souris dans sa trompe pour signifier au jeune homme qu'il ne faut pas mépriser ses concurrents, comme il le fait, car ceux qui semblent plus faibles s'avèrent parfois supérieurs ; le "túmulo» de l'oubli auquel le conduit la " Calle de la Ausencia " et le grand cyprès, symbole de mort sur le tronc duquel est gravée une croix p. 256), tous ces emblèmes donc, sont enchâssés dans le labyrinthe floral de Narcisa, lui-même emblème de l'œuvre comme on l'a vu précédemment. Or, l'enchâssement ou la mise en abyme est un procédé qui est à l'ouvre à tous les niveaux dans la structure des Cigarrales de Toledo, au niveau des récits, des genres et du système « cigarral ».

Finalement, dernier point qui vient corroborer l'idée que le labyrinthe floral de Narcisa est l'emblème de la structure des Cigarrales de Toledo, la symbolique des chiffres que l'on y trouve. En effet, la construction du labyrinthe est fondée sur une combinaison des chiffres 2 et $3: 2$ portes qui ouvrent chacune sur 3 chemins ; 6 concurrents, 6 "doncellas » et 6 pages pour accueillir les jeunes nobles à la fin du divertissement. Or, ces deux chiffres sont complémentaires d'un point de vue symbolique : le 2 est le nombre de la Terre, le 3 celui du Ciel. Le « labyrinthe d'arbres et de fleurs" réunirait ainsi la terre (cf. le jardin) et le ciel (cf. la dimension spirituelle et morale des emblèmes). Le 2 est symbole de toutes les ambivalences et des dédoublements alors que le 3 pour les chrétiens symbolise la perfection de l'Unité divine $^{12}$. Mais surtout, ils sont la somme $(3+2)$ des 5 cigarrales de l'œuvre ou le produit $(3 \times 2)$ des 6 parties de l'œuvre : l'Introduction très longue suivie des 5 cigarrales-chapitres. Ainsi le labyrinthe du cigarral II représente à mon sens l'emblème de la structure de l'œuvre tirsienne. Mais nous allons voir dans un deuxième temps que ce Bois d'Amour et le "Château de la Prétention d'Amour " constituent un divertissement qui emblématise ou allégorise toutes les thématiques présentes dans l'œuvre de Tirso.

11. Cf. Dictionnaire des symboles, ed. cit.

12. Cf. Dictionnaire des symboles, ed. cit. 


\author{
II. LE JEU DU LABYRINTHE, \\ EMBLÈME DES THÉMATIQUES DE L'GEUVRE
}

\title{
II.1 Le divertissement et le plaisir
}

Le labyrinthe de Narcisa s'inscrit bien sûr dans la longue chaîne des divertissements qui jalonnent les Cigarrales de Toledo : à partir du cigarral II, chaque roi ou reine de chaque cigarral propose à ses hôtes deux divertissements séparés par le repas de midi et la sieste. C'est ainsi que les invités du cigarral I assistent à la représentation d'une comedia, ceux du cigarral II prennent part au jeu du labyrinthe de Narcisa puis écoutent la Fábula de Siringa y Pan. Dans le cigarral III, véritable cœur du récit tirsien, ce sont les récits de don Juan de Salcedo puis de Dionisia qui servent de divertissements entre le matin et l'après-midi. Au cigarral IV, une récitation de poèmes puis la représentation de la comedia Cómo han de ser los amigos constituent les divertissements. Enfin, dans le dernier cigarral, les personnages écoutent la Novela de los tres maridos burlados le matin puis assistent à la comedia El celoso prudente. Le labyrinthe floral et le château de Narcisa ne sont donc qu'un divertissement de plus. Mais il est remarquable que le texte dans notre passage insiste avec force sur l'idée de divertissement et de plaisir qui en découle, notamment par la Letra qui précède le jeu du labyrinthe. Cette Letra correspond à une véritable programmation du plaisir et elle place sans équivoque le labyrinthe de Narcisa sous le signe du plaisir et du jeu. En effet, elle est fondée sur la dichotomie placer / pesar ainsi que la valorisation du premier terme au détriment du second comme l'indiquent clairement le refrain : "¿Y el pesar? / „No ha lugar!" (p. 234) et encore ces vers : " No ha convidado Narcisa / en su Bosque de Amor / al llanto, pena y dolor, / sino al gusto, fiesta y risa" (p. 235). Citons aussi la première strophe qui nous fait entrer résolument dans le royaume du plaisir et du jeu : " $A l$ Bosque de Amor esfera / solamente podrá entrar / el placer que el gusto espera" ou encore les deux premiers vers de la deuxième strophe : "Solamente ofrece entrada / al regocijo esta puerta" (p. 235). De plus, cette Letra est chantée par "un hermoso niño, figurado en él el Placer" (p. 234). Autrement dit, Tirso de Molina introduit le divertissement proposé par Narcisa à ses hôtes par une véritable allégorie du Plaisir et mêle ainsi savamment dès le départ, allégorie, emblème et jeu ou divertissement.

On remarque également que le lexique du plaisir est abondant dès cette Letra qui programme le plaisir que doit procurer le jeu du labyrinthe et 
du Château et tout au long du divertissement. Ainsi reviennent sans cesse les termes entretenimiento, entretener, regocijo, placer, regocijadamente, gusto, contento, alegre, reir, risueña, deleitosa pour bien signifier que les épreuves du labyrinthe doivent être prises comme un jeu et n'ont d'autre prétention que de distraire les hôtes de Narcisa même si, comme on l'a vu précédemment, chaque concurrent reçoit une leçon morale en traversant ces épreuves. On retrouve alors de façon très claire le grand précepte horatien que Tirso de Molina a repris à son compte : le "delectare ac prodesse " ou, en langue tirsienne, le "Deleytar aprovechando ». Or, j'avais tenté de montrer dans la dernière partie de ma thèse intitulée: "La Poétique tirsienne ou la démonstration du "Deleytar aprovechando" " que les Cigarrales de Toledo sont une Poétique qui se fonde essentiellement sur ce principe du plaisir, du divertissement comme moyen de transmettre un savoir, un enseignement moral, poétique ou littéraire. La littérature n'est alors pour Tirso qu'une façon de joindre l'utile à l'agréable pour paraphraser la formule d'Horace. C'est pourquoi l'insistance du texte tirsien sur le thème du divertissement et du plaisir dans le passage que nous étudions n'est pas anodine : elle signifie, dans un jeu intellectuel et littéraire, que Tirso souhaite faire de ce jeu allégorique du labyrinthe, le Bois et le Château d'Amour, la représentation emblématique du thème structurant du divertissement dans son œuvre. Or, je l'ai dit : le divertissement est un fil thématique et structurant qui court tout au long des Cigarrales de Toledo et qui prend véritablement sa dimension systématique à partir du cigarral II, précisément à partir de l'amusement du labyrinthe de Narcisa.

De plus, au plaisir des yeux, de l'intellect et de l'ouïe ${ }^{13}$ que suppose le divertissement proposé par Narcisa est associé, comme dans chaque cigarral à venir, celui de la bouche et de la bonne chère. En effet, comme dans chacun des trois cigarrales suivants, les divertissements du matin et de l'après-midi sont séparés par un magnifique repas offert par le roi ou la reine du cigarral. Mais ici, Tirso atteint un degré de symbiose entre les différents plaisirs qu'il n'égalera pas par la suite. En effet, le divertissement du Bois et du Château d'Amour n'est pas simplement suivi d'un festin, il se dilue littéralement en un festin car le château que les prétendants d'amour ont finalement pris d'assaut se métamorphose sous les yeux ébahis des personnages en une table de banquet magnifiquement dressée. Je cite le texte :

... dieron de repente en tierra los cuatro lienzos de la muralla, fábrica de toda aquella vistosa máquina, sirviendo, los que primero fueron muros

13. Cf. le chant de la Letra et la musique qui accompagne l'attaque du Château par les prétendants à l'amour. 
defensivos, agora de curiosas alfombras y quedando en su primero ser el chapitel que primero fue piramidal extremo del CASTILLO, y agora bizarra coronación de un cenador apetitoso, debajo del cual vieron en cruz cuatro prolongadas mesas, llenas de diversos manjares... (p. 258).

La métamorphose est aussi soudaine que brutale (" de repente») provoquant la chute des assaillants du Château qui étaient parvenus à la hauteur des créneaux mais une chute prévue ("los derribó en el foso - que en vez de fajina tosca estaba cubierta de blandas yerbas y regaladas flores" (p. 258)) et nécessaire pour ouvrir enfin le Château-forteresse ("la defendida fortaleza"; " el florido alcázar "; " entrada y salida del Bosque y Fortaleza ") aux plaisirs de l'amour et $\mathrm{du}$ banquet. On remarque que les prétendants à l'amour tombent dans des fossés de fleurs, symboles de beauté, de renouveau, d'amour et de douceur et que le mot-clé apparaît dans cette description de la métamorphose du Château en table de banquet fastueux : le mot "máquina " qui dit la mise en scène et la théâtralité de ce divertissement mais aussi la composition artificieuse et artistique de tout le Bois d'Amour.

L'art consommé de l'artifice dont a fait preuve la créatrice du jeu du labyrinthe, est maintes fois mis en évidence et en particulier dès la phrase d'introduction au divertissement que j'ai citée au début de mon étude : "No se habia descuidado la solícita dama pues ayudada de sus deudos [...] habia, en la entrada del celebrado Cigarral, dispuesto un enramado laberinto de árboles y flores... ". Puis il est question du génie de Narcisa comparée à Dédale, de "la industriosa Narcisa " (p. 234), de "la discreta Narcisa " (p. 247), de la "sutileza de la autora" du labyrinthe (p. 239). À diverses reprises, reviennent les louanges de l'esprit inventif et ingénieux de la reine du cigarral. Par ailleurs, l'adjectif artificioso qualifie souvent les parties du labyrinthe conçu par le Dédale tirsien et les mots artificio et arquitectura sont utilisés à plusieurs reprises pour désigner le Bois d'Amour : "artificioso camino" (p. 239), "artificioso bosque" (p. 234), "la artificiosa maraña " (p. 246), "la artificiosa Selva" (p. 246), " el artificio» (p. 248), " un sutil artificio" (p. 258), "la artificiosa armonía " (p. 259) etc. Or, ces termes d'art et d'artifice sont des termes-clés pour le dramaturge baroque qu'est Tirso de Molina. Ils sous-tendent en grande partie sa conception de la littérature qui doit régaler l'esprit par de subtiles inventions et d'ingénieuses créations. Ainsi, ce Bois allégorique d'Amour et ce "Château de la Prétention d'Amour » sont avant tout des architectures éphémères, des "machines " qui sont conçues comme de véritables spectacles à la fois pour les personnages de l'histoire et pour le lecteur, lesquels partagent un plaisir intellectuel dû en grande partie à l'artifice et à l'ingéniosité de la construction, de l'invention. De ce 
point de vue-là aussi, le jeu du labyrinthe est l'emblème des thématiques du divertissement, du plaisir et de l'artifice que développe toute l'œuvre, véritable poétique du "deleytar aprovechando ". Mais le divertissement de Narcisa est aussi et surtout fondé sur le thème de l'amour, l'amour à atteindre, l'amour à conquérir, qui est lui-même un thème structurant dans les Cigarrales de Toledo. Je voudrais donc terminer par une rapide analyse de cette thématique, emblématisée dans le cigarral II.

\section{II.2 L'amour emblématisé}

On constate en fait que l'amour est présent à tous les niveaux du jeu du labyrinthe sous diverses formes. Ainsi, il apparaît dans les noms des différents chemins empruntés par les six prétendants à l'amour, noms qui eux-mêmes développent le nom de chaque porte du labyrinthe. La porte de la confiance se décline en trois sortes de confiances qui toutes ont un rapport avec l'amour et la conquête amoureuse : "confianza de amor ", " estimación de sus servicios" et "menosprecio de sus competidores". La porte de la méfiance ou du soupçon se décline, quant à elle, sous trois formes de méfiance : "temor", "poca satisfacción de sí " et "celos en duda ". Par conséquent, le jeu du labyrinthe met en concurrence les deux attitudes opposées de tout homme amoureux souhaitant conquérir l'amour de l'élue de son cour. De plus, sur plusieurs allées du labyrinthe, apparaissent des références à l'amour et/ou à ses corollaires. Ainsi, par exemple, le chemin de don Alonso se termine par l'Allée de l'Absence (on comprend l'absence de l'être aimé) qui débouche sur une place où se trouve le "Túmulo del olvido" sur lequel est représentée une femme apparemment morte : "La voluntad de tu dama " explique une devise. Don Alonso doit comprendre que sa suffisance peut avoir pour conséquence l'oubli de dońa Leocadia, sa dame, et son absence comme l'indique une légende en forme d'épitaphe au pied de la croix sur le grand cyprès (symbole de mort lié au culte de Pluton, dieu des enfers chez les Romains) : "Aqui mató la ausencia presumida una voluntad amante. Rueguen a Dios por ella" (p. 256). Sur le parcours de don Fernando, celui "del temor ", différentes voies s'offrent à lui plus ou moins larges et correspondant aux divers effets de la Crainte que l'on peut aussi considérer comme les corollaires de l'amour malheureux dû précisément à la crainte et à la pusillanimité de l'amant : Désespoir, Vengeance, Colère, Courroux, Impatience. Au bout du chemin de don Melchor, c'est le dieu de l'Amour Cupidon qui apparaît portant dans sa main droite une couronne de chêne, symbole de force et de robustesse. Enfin, l'amour est aussi présent 
sous la forme symbolique des fleurs telles que le myrte, arbuste consacré à Vénus, la déesse de l'amour, et la rose, fleur consacrée aussi à Aphrodite et qui symbolise la coupe de vie, l'âme, le cour et l'amour. Or, dès le début, le texte insiste sur le fait que les trois allées sur lesquelles s'ouvrent les deux portes du labyrinthe floral sont bordées de myrte ("...en cada parte tres calles hechas de murta, arrayán y otras yerbas olorosas " p. 239) et que l'arche de l'entrée du labyrinthe est faite de roses entre autres fleurs (p. 234). Puis, tout au long du divertissement, les références au myrte et à la rose sont abondantes. L'on voit donc que l'amour, qui d'ailleurs est écrit avec un " $A$ » majuscule dans les différentes expressions qui évoquent le jeu du labyrinthe ("Bosque de Amor ", "Castillo de la Pretensión de Amor "), est le thème prépondérant du passage comme il l'est de toute l'œuvre - je vais y revenir mais n'oublions pas dès à présent que le point de départ du récit tirsien prend prétexte de trois noces qui ont lieu à Tolède et sont l'occasion de réjouissances.

Mais la preuve la plus éclatante de cette prépondérance thématique de l'amour est, c'est une évidence, le passage consacré au château-forteresse dans lequel se sont enfermées les dames des six galants. Lorsque ces derniers se préparent à prendre d'assaut le château, brandissant drapeaux et étendards, en haut des murailles apparaissent les dames portant des écriteaux sur leurs poitrines, représentant ainsi les passions de la jalousie, de l'amour, de la présomption, de la crainte et de la confiance : "representaban las encontradas pasiones de los celos, amor, presunciones, temores y confianzas " (p. 256). A l'intérieur du jeu du labyrinthe, ce château, on l'a vu, représente le centre du labyrinthe, c'est-à-dire le but ultime de la quête amoureuse que symbolisent les différents parcours des six prétendants d'amour. Si l'on reprend la symbolique du château, puisque tout dans ce passage des Cigarrales est à prendre au sens symbolique ou allégorique, il est un lieu qui symbolise la protection et qui protège la transcendance du spirituel mais aussi qui symbolise la conjonction des désirs comme nous le rappellent la poésie des troubadours et les poèmes d'amour de Jorge Manrique ${ }^{14}$. On peut alors considérer que ce "Château de la Prétention d'Amour " est l'emblème du thème principal de l'œuvre tirsienne car tous les parcours initiatiques des six galants représentent ceux des personnages du récit des Cigarrales de Toledo qui tous, tant au niveau principal que secondaire du récit, sont en quête d'amour ou à la recherche de l'être aimé. Par conséquent, ce que font les six prétendants à l'amour sous la forme d'un jeu (la quête amoureuse par le cheminement dans le labyrinthe puis la conquête amoureuse par l'attaque

14. Par exemple le Castillo de amor, in Jorge Manrique, Poesía, ed. De Jesús-Manuel Alda Tesán, Cátedra, Madrid, 1993, p. 93-97. 
du château) n'est à tout prendre que ce que font, à plus grande échelle en quelque sorte, notamment le couple principal don Juan de Salcedo et Lisida, ainsi que le couple de Catalans Dionisia et don Dalmao. De la même façon, la grande affaire des personnages des comedias insérées dans l'œuvre est l'amour ${ }^{15}$. En effet, si tous les personnages principaux du récit tirsien sont des pèlerins d'amour, les six prétendants du jeu du labyrinthe sont aussi des pèlerins d'amour qui font le voyage initiatique de ce labyrinthe et tentent d'atteindre la félicité amoureuse en conquérant le château où sont enfermées leurs dames, qu'ils doivent libérer. Ils emblématisent par leur action dans le divertissement de Narcisa le pèlerinage d'amour que font les personnages principaux dans le bassin méditerranéen entre Tolède, Barcelone et Naples. Ainsi, pour le héros tirsien, don Juan de Salcedo, ce château de verdure du cigarral II représente le centre de sa quête et de son périple méditerranéen : Tolède où réside sa bien-aimée Lisida qu'il retrouve au début du récit après deux ans et demi d'absence.

Il faut ajouter pour finir que les six participants au jeu du labyrinthe, en recevant un enseignement moral et en subissant une métamorphose, comme on l'a vu précédemment, passent de l'engaño au desengaño (c'est littéralement vrai dans le cas de don Suero). Or, j'ai montré dans ma thèse que les personnages de l'œuvre de Tirso subissent aussi ce passage de l'engaño au desengaño et que cette thématique est absolument structurante tant dans le récit que dans les comedias et la nouvelle du cigarral V. En effet, l'engaño déclenche le processus de dégradation / amélioration par lequel fonctionne tout récit et le desengaño permet de le mener à son terme. Ainsi, tous les personnages se croient trompés, sont victimes de leurs sens abusés, utilisent des déguisements et des masques et finissent toujours par être détrompés. Je ne peux pas rentrer dans les détails mais il est remarquable que le thème du paraître et de l'illusion soit très présent dans le premier divertissement du cigarral II. Le texte ne cesse de souligner le caractère factice de toute l'architecture florale et éphémère du divertissement. Ainsi, de nombreuses expressions telles que "engaños del pincel ", "encantamientos ", "fingido encantamento " (p. 238), "al parecer" ou " en la apariencia " (dans des phrases du type : "yerbas al parecer macilentas" p. 241, "al parecer de mármol " p. 242, "en la apariencia de bronce ", ou bien encore "recelando nuevos engaños " p. 248), "el engañado " pour désigner un des concurrents, "la engañosa cama» (p. 259), l'emploi fréquent du verbe parecer (parecía de piedra, parecía de alabastro p. 254) etc. traduisent le fait que Narcisa a fait

15. Je renvoie à ma thèse dans laquelle j'ai montré que l'amour est le moteur de l'action des personnages du récit tirsien. 
monter une illusion, un spectacle qui trompe les sens mais pour mieux détromper les spectateurs, les lecteurs et les acteurs du divertissement. D'ailleurs, la fábrica de toda aquella vistosa máquina (p. 258) s'effondre à la fin comme pour mieux convaincre le lecteur et les personnages du caractère trompeur, illusoire et éphémère de tout le labyrinthe et du château. On comprend alors que tout cela n'est qu'un jeu mais un jeu qui permet de faire comprendre à ses participants leurs erreurs et au lecteur de s'interroger sur l'apparence et l'illusion. Le thème de "l'aberration des sens " pour reprendre l'expression d'André Nougué ${ }^{16}$ au sujet du théâtre tirsien, est bien ici dans notre passage littéralement emblématisé par cette "machine ", cette construction splendide et éphémère du "Château de la Prétention d'Amour ».

Ainsi donc, ce jardin de Narcisa dans le cigarral II constitue bien un modèle d'artifice et de trompe-l'œil et si, comme je l'ai montré dans ma thèse, l'image du labyrinthe est le symbole récurrent dans l'œuvre de la structure des récits et comedias fondée sur la complication et l'engaño, alors le divertissement labyrinthique de Narcisa est le symbole par excellence et même plus exactement l'emblème de tous les jardins tirsiens, de ces Cigarrales de Toledo, dédale fictionnel et narratif, où nous entraine leur auteur tout comme Narcisa invite ses hôtes à parcourir les allées tortueuses et emblématiques de son labyrinthe d'arbres et de fleurs.

16. André Nougué, «Le thème de l'aberration des sens dans le théâtre de Tirso de Molina. Une source possible ", Bulletin Hispanique, Tome LVIII, 1956, p. 23-35. 